\title{
Neumopatía aspirativa iatrogénica
}

\author{
DRES.: R. BURDACH *, D. MIRKIN **, A VARAS *, L. BOZA *, E. CORTES *, C. BARROS *, C. \\ PINO *, H. GRAVET*.
}

\begin{abstract}
La importancia de la Etiología aspirativa en la génesis de Bronconeumopatías crónicas destaca en el niño menor, debido a mecanismos patogénicos atribuibles a inmadurez neuromuscular faríngea $(6,9)$ o a insuficiencia gastroesofágica $(4,7,10,11,14)$. La calidad del material aspirado puede determinar un daño bronquial o tisular severo, que suele ser subestimado por el médico al indicar tratamientos a base de substancias que representan un peligro potencial en este sentido.
\end{abstract}

La frecuencia de las Neumopatías aspirativas en el lactante menor es señalada desde hace tiempo en la literatura pediátrica. Sin embargo, la relación etiopatogénica del mecanismo aspirativo $(1,10)$ con bronconeumopatías crónicas, que se atribuían a infecciones, alteraciones inmunoalérgicas o a contaminación ambiental, constituye tema de publicaciones recientes $(4,7,11)$. En ellas se destaca la importancia más sutil del examen radiológico del tubo digestivo, que permite demostrar, por una parte la incoordinación faríngea en el lactante menor y por otra, la existencia del reflujo gastroesofágico en casos de insuficiencia hiatal $(2,14,15))$ con o sin ectopia gástrica en lactantes, pre-escolares y aun en niños mayores $(10,11)$. La aspiración se explica, en decúbito supino, por ausencia del tono muscular a nivel de la unión gastroesofágica, la abertura del ángulo de His, falta de oclusión laríngea e ingreso del contenido gástrico desde la faringe a la tráquea en forma de un microaerosol (7).

- Departamento de Enfrmedades Broncopulmonares. Hospital M.

* Arriarán.
La biopsia pulmonar, como método diagnóstico, en los casos de Neumopatías crónicas de etiología no precisable mediante los exámenes habituales, permite detectar muchas veces el agente causal, conduciendo a la adopción de medidas preventivas y, eventualmente, al seguimiento de la evolutividad de las lesiones.

El presente trabajo tiene por objeto, dar a conocer la importancia de algunas sustancias de uso terapéutico habitual en la génesis de Neumopatías crónicas del lactante.

\section{Casufstica}

Caso No 1:

R. M. D. Obs. N 9259284 . Lactante de 3 meses, ingresado el 4-V - 73 al Hospital M. Arriarán con el antecedente de constipación, que motivó la indicación de vaselina líquida administrada durante casi 2 meses en forma continuada. Debido a la existencia de vómitos y regurgitaciones frecuentes, se solicitó un tránsito digestivo, que demostró una insuficiencia gastro - esofágica. Al ingreso llamó la atención su retardo ponderal $(5.050 \mathrm{~g}$.$) , polipnea permanente \left(100-120 \mathrm{x}^{\prime}\right)$, asimetría torácica con abombamiento del H. D., aumento del diámetro AP, retracción subcostal, hipersonoridad bilateral y broncofonía del $1 / 3$ medio derecho. La radiografía de tórax reveló: sombra difusa de condensación del tercio superior y medio derechos, con enfisema retroesternal (Fig. 1). El tratamiento antibiótico (Penicilina - Gentamicina) y esteroidal (Prednisona) durante 20 días no modificó el cuadro clínico-radiológico, que se mantuvo durante 2 meses sin variaciones. Los exámenes complementarios: Astrup, hematológicos, electrolitos del sudor, cuantificación de inmunoglobulinas, test de precipitinas y ECG, no aportaron nada de especial. 


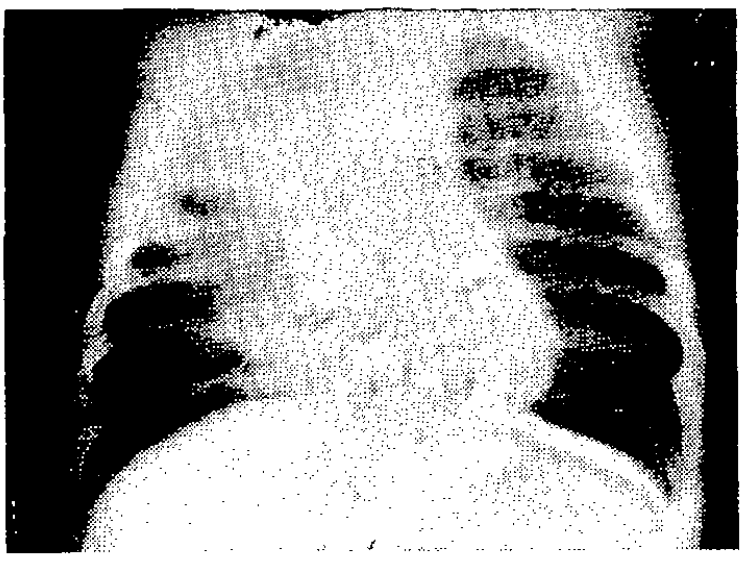

1. Radiografía frontal. La sombra de condensación del tercio $\mathrm{su}^{-}$erior reveia límites más neios en el borde inferjor.

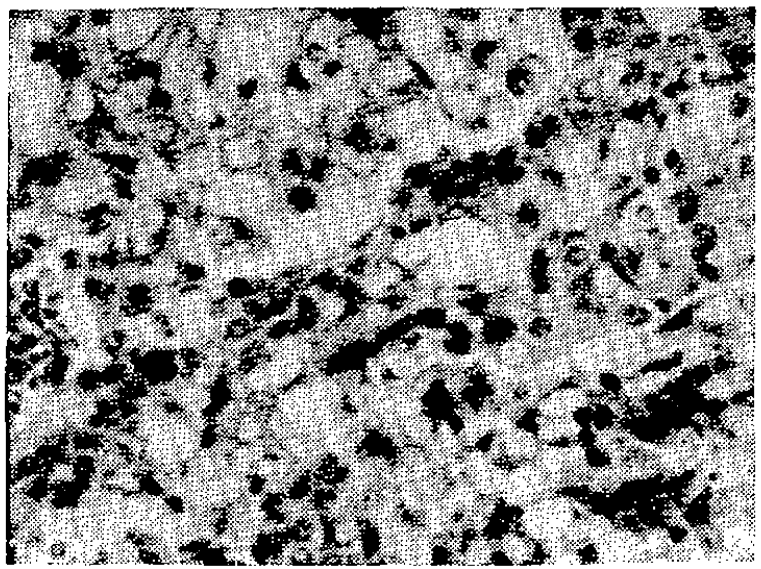

2. Microfotograf́a. Se observa reemplazo de la estructura pulmonar nor macrófagos de grandes vacuolas que ocupan cl intersticio y especlos respiratorios. Hematoxilina - cosina. $40 \mathrm{X}$.

Se planteó la hipótesis diagnóstica de Neumonía lipoidea y a los 5 meses de edad se practicó la biopsia pulmonar a cielo abierto del L. S. D. La superficie pleural del trozo resecado $(25 \times 15 \times 8 \mathrm{~mm}$.) exhibía áreas condensadas de color violáceo, congestión y focos de hemorragia. El examen microscópico demostró extensas áreas de condensación, en que la estructura alvcolar había sido reemplazada por células histiocitarias, cuyo citoplasma estaba constituido por una gian vacuola negativa y el núcleo desplazado hacia en bords. Existía moderada reacción fibrosa sin formación de células gigantes y algunos bronquiolos, cuyo lumen contonia abundantes células lipofágicas (Fig. 2).

El niño siguió posteriormentc en control ambulato. rio hasta la actualidad ( 1 año 7 meses de edad), sometido a terapia esteroidal discontinua durante 9 meses. En sus controles se pudo apreciar un progreso ponde. ral de 4400 g., normalización de la auscultación pulmonar ( $8^{\circ}$ mes) pero persistencia de la polipnea ( $\mathrm{s}^{3} \mathrm{~b}$ re $60 \mathrm{x}$ ) y de alteraciones radiológicas representadas por una sombra densa de borde externo convexo a nivel de la región hiliar derecha.

\section{Caso No 2:}

P. D. G. Obs. N 256313. Lactante de 1 año, enviado desde provincia (Pto. Montt) con el diagnóstico de Neumoniiis crónica. Desde los 6 meses de edad, a continuación de un cuadro diarreico, tratado con medidas dietéticas, que incluian Arobón (R), comenzó a presentar tos emetizante, disnea, fiebre intermitente y estacionamiento de la curva ponderal. Estuvo hospitalizado durante 45 dias, tratado con antibióticos y ambiente húmedo (Croupette) sin experimentar mejoría. El estudio radiológico demostró sombras difusas parahiliares y basales bilaterales (Fig. 9). Ante la persistencia de la sintomatología clínica y radiológica, se decidió su traslado, ingresando al Depto. de Broncopulmonares del Hospital M. Arriarán el 12-I - 73. Se comprobó un lactante en deficiente estado nutritivo $(6.180 \mathrm{~g}$.) con polipnea $\left(60 x^{\prime}\right)$, aumento del diámtero AP del tórax, retracción subcostal, broncofonía del tercio medio derecho y algunos estertores subcrepitantes en los $2 / 3$ inferiores del H. D. Se practicaron exámenes radiológicos del tórax, esofagograma, Astrup, electrolitos del sudor y test de precipitinas a leche de vaca, de los cuales sólo destacaban las alteraciones radiológicas anteriormente señaladas.

Dada la evolución clínico - radiológica de curso crónico, se decidió efectuar la biopsia pulmonar (marzo 73) y se extrajo una muestra de L. M. de $12 \times 8 \times 3 \mathrm{~mm}$., en cuyo aspecto macroscópico destacaba un área condensada de $4 \mathrm{~mm}$. de diámtro de aspecto hemorrágico. El cxamen microscópico reveló focos de condensación con acúmulos de linfocitos que formaban verdaderos folículos linfáticos y a distancia corpúsculos de material muy denso ópticamente de color pardo refringente (Fig. 4), que en partes constituian granulomas con inclusión de células gigantes multinucleadas de tipo cuerpo extraño en relación a estos corpúsculos, de tamaño irregular de hasta 500 micrones de diámetro, de bordes rectos y ángulos agudos (Fig. 5). Además existfan

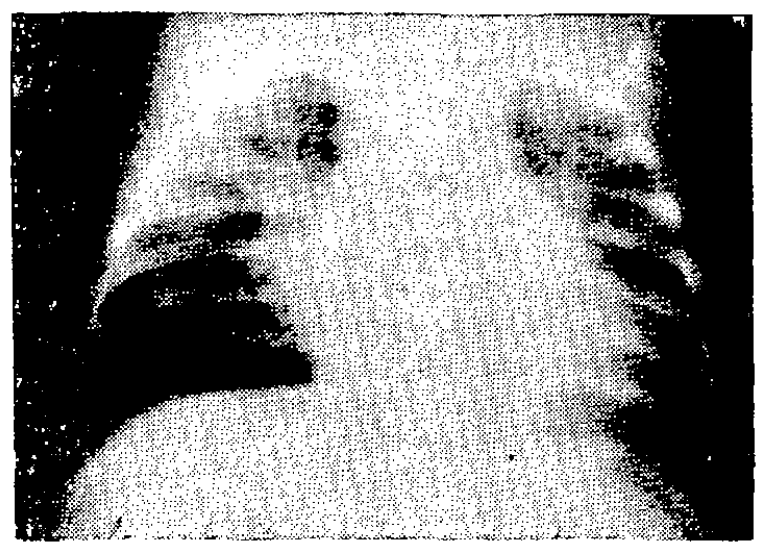

3. Radlofragía frontal. Sombras difusas parahillares bilaterales. 
zonas de enfisema y alvéolos que contenian histiocitos grasos, de citoplasma finamente reticulado.

Siguió control ambulatorio, sometido a terapia esteroidal discontinua y Sulfaprofilaxis durante 10 meses. La evolución se caracterizó hasta el momento del alta (edad: 2 años 2 meses) por persistencia de polipnea (60 $\mathrm{x}^{\prime}$ ), normalización de la auscultación pulmonar, pero persistencia de alteraciones radiológicas, representadas por aumento de densidad hiliar a derecha y sombras retículocordonales en el campo pulmonar del mismo lado.

\section{Caso No 3:}

H. J. F. Obs. N: 191518. Lactante de 1 año 1 mes, que ingresó al Hospital M. Arriarán el 29 - II - 1968 por un cuadro diarreico, tratado con mezcla dietética que contenía Arobón ( $R$ ), apareciendo luego reacción febril, tos y disnea de 7 días de evolución de pre - ingreso.

Se comprobó un lactante grave, con déficit ponderal

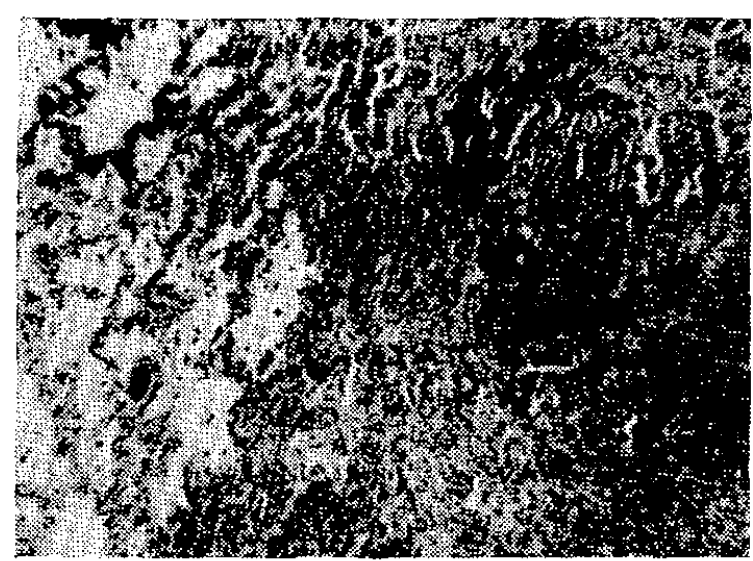

4. Microfotografía. Formación de estructura Jinfoidea (L) en área de condensación crónica y próxima a ésta, partícula de Ceratonfa (flecha) Hematoxilina-cosina $6,3 \mathrm{X}$.

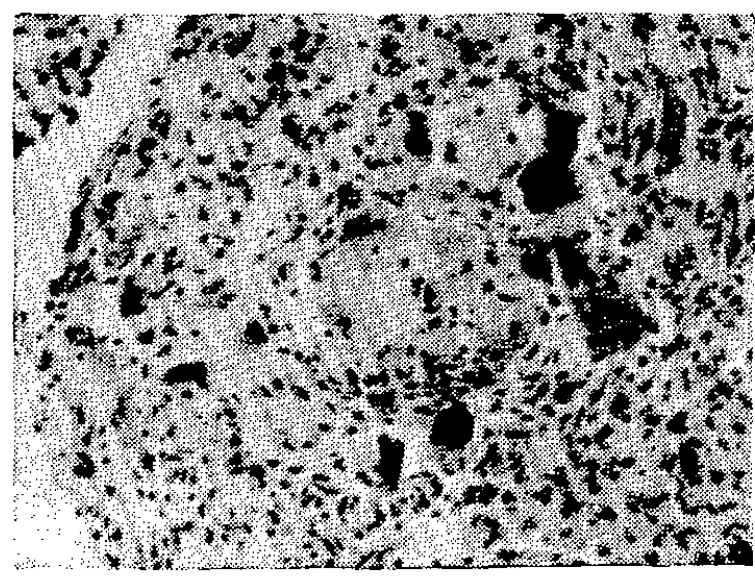

5. Microfotografia. En el centro, células gigantes multinticleadas $y$ en relactón con éstas varias partf́culas de Ceratonia. Hema. toxilina - cosina $16 \mathrm{X}$.
(6400 g.), polipnea (52 $\mathrm{x}^{\prime}$ ) signología bronquial obstruc. tiva difusa $\mathrm{y}$ hepatomegalia.

La radiografía de tórax reveló sombras de condensación en el LSD, LM y ambos inferiores, con descenso del diafragma.

A pesar del tratamiento antibiótico, inicialmente a base de Penicilina-Kanamicina y luego Meticilina, el cuadro clínico-radiológico no se modificó sustancialmente, evolucionando el sindrome bronquial obstructivo con remisiones parciales y nuevas exacerbaciones. Durante 45 días recibió terapia esteroidal a base de Prednisona sin influencia favorable sobre la sintomatología descrita.

Los exámenes dirigidos a precisar la etiología: elec. trolitos dol sudor, investigación de hemosiderófagos mediante punción pulmonar, test de precipitinas a proteínas de leche de vaca e intradermoreacción a leche de vaca, fueron normales.

En estas circunstancias se decidió efectuar la biopsia pulmonar (4\% mes) y se extrajo una muestra del LM y LSD mediante la técnica a cielo abierto. Macroscópicamente se comprobó el LM de aspecto nodular con limitación de su expansión. Microscópicamente se observaron áreas de condensación con engrosamiento de tabiques interlobulillares con fibrosis e infiltración inllamatoria crónica; células gigantes que contenían corpúsculos de color café y otras con material extraño eosinófilo, rodeadas de delgadas láminas tenuemente basófilas.

Dado de alta después de $41 / 2$ meses, siguió con persistencia de signología bronquial obstructiva y alteraciones radiológicas, consistentes en sombras difusas parabiliares y paratraqueales derechas, que se extienden hacia los $2 / 3$ inferiores del mismo lado y sombras en región parahiliar y basal izquierda.

Se rehospitalizó a los 13 meses de control durante 1 mes, para someterlo a terapia con DEMSO y corticuides (Aradix R) sin observarse modificación importante de la sintomatologia.

El control posterior continuó hasta los 3 años de edad, manteniéndose en iguales condiciones.

Comentario: Si bien el cuadro de la Neumopatía aspirativa es muy conocido en la literatura pediátrica (1), su mecanismo etiopatogénino y la posibilidad de generar lesiones irreversibles y posiblemente progresivas, ha sido mejor investigado a través de la mayor difusión de nuevas técnicas de explnración. El hecho de poder detectar la aspiración mediante la adecuada interpretacion del examen radiológico del tracto digestivo, ha permitido demostrar la relación de causa a efecto en la génesis de múltiples cuadros respiratorios crónicos o recidivantes. Este mecanismo puede obedecer a alteraciones del segmento digestivo superior representados por: incoordinación farígea en R. N., prematuros y lactantes menores; a una inma- 
durez neuromuscular, a daños neurológicos o a una disautonomía congénita debida a la actividad anormal del músculo cricofaríngeo. En la literatura se menciona la incoordinación faríngea en un $13 \%$ de $\mathrm{R}$. N. normales (6).

Además destaca como factor importante la insuficiencia gastroesofágica con o sin ectopía gástrica, que puede observarse a edades más avanzadas siendo responsable de sintomatología respiratoria crónica en proporciones variables, que diversas publicaciones la hacen fluctuar entre un $20-75 \%$ $(4,7,10)$.

La demostración de la aspiración del contenido gástrico, que no se exterioriza por vómitos o regurgitaciones, es difícil, porque no ha sido posible confirmar la presencia de jugo gástrico en la tráquea, y sólo ocasionalmente el examen fluoroscópico ha permitido apreciar el paso del medio de contraste hacia las vías respiratorias.

En cambio, la inmadurez neuromuscular en el lactante menor vomitador ha podido detectarse al comprobar el ingreso del medio de contraste de tensión superficial similar al de la leche, al árbol traqueobronquial (9). La confirmación de que el material aspirado es el causante de la Neumopatía, la obtenemos mediante la biopsia pulmonar. La inespecificidad de sus manifestaciones clínico-radiológicas y la dificultad técnica de realizar algunos exámenes funcionales en el niño menor, puede superarse a través del estudio morfológico que permite afinar nuestros conocimientos referentes a la etiología y pronóstico de estas afecciones. Constituye un método diagnóstico de innegable valor en presencia de Neumopatías catalogadas vagamente como: Enfisema, Neumonitis o Fibrosis localizadas o difusas, de carácter persistente o progresivo.

Hemos preferido la biopsia a cielo abierto, porque reúne las ventajas de su bajo riesgo, al evitar complicaciones como Neumotórax o hemorragias que suelen ocurrir con otros procedimientos y permite bajo control visual la obtención de una muestra suficiente del lugar más indicado. Mediante este examen pueden pesquisarse lesiones iniciales, que antiguamente sólo se conocían en su etapa final y descubrir etiologías hasta el momento no sospechadas entre nosotros, como ocurrió con dos de nuestros casos.

La Neumonía lipoidea correspondiente a reacciones fibrosas intersticiales asociadas con la presencia de lípidos intraalveolares, ha sido reconocida desde hace largo tiempo $(3,5,12)$.
El lípido no tiene que ser necesariamente inhalado, sino que puede ser liberado en el propio pulmón, como ocurre con mayor frecuencia en procesos que causan destrucción tisular, tales como abscesos, neumonías no resueltas o tumores. En estos ejemplos la Neumonía lipoidea endógena no tiene localización determinada por gravitación y microscópicamente los lípidos tienden a estar finamente dispersos en el citoplasma de los macrófagos que adquieren aspectos espumoso. La Neumonía lipoidea exógena, como la que se presenta en nuestro caso, se distingue en que las vacuolas intracitoplasmáticas en los macrófagos, son grandes y desplazan a los núcleos.

La naturaleza del irritante inhalado condiciona el monto y velocidad de los procesos de fibrosis. Los menos ofensivos son el aceite de oliva y los aceites vegetales neutros usados como vehículos de medios de contraste. Mucho más irritante es el aceite mineral, a pesar de ser químicamente inerte. Es emulsionado y fagocitado, pero el residuo induce lentamente a la fibrosis.

Los lípidos de origen animal tienden a descomponerse y son convertidos por la acción de las lipasas en ácidos grasos irritantes, que estimulan una inflamación bastante severa y fibrosis de progresión más rápida. En los lactantes, confinados al decúbito supino, las lesiones predominan en los lóbulos superiores, especialmente el derecho. La lesión más común es un proceso difuso, que comienza como focos diseminados, que luego se hacen confluentes. Gran parte o todo un lóbulo puede consolidarse y adquirir junto con el aspecto sólido una superficie amarillenta pálida.

El llamado granuloma lipoide puede presentarse como un área bien localizada, de forma redondeada, como tal vez lo demuestra la evolución radiológica del caso presentado. En este paciente, la aspiración de vaselina, de causa iatrogénica dio lugar a un cuadro severo con persistencia de alteraciones radiológicas hasta los 17 meses de control, y de polipnea en reposo, que demostró una hiperventilación crónica, debido al bloqueo de territorios alveolares inutilizados.

$\mathrm{Su}$ patogenia obedeció probablemente al reflujo gastro-esofágico. Esto obliga a reconsiderar la actitud del pediatra ante cuadros similares, en los que un motivo banal, como la constipación, puede condicionar mediante el uso de preparados aceitosos, la instalación de una Neumopatía crónica e irreversible de difícil manejo. Debería inducirnos a eliminar del arsenal terapéutico a agentes poten- 
cialmente nocivos, dada la gran frecuencia de la aspiración demostrada en el lactante menor.

En la larga lista de substancias, que en forma de partículas pueden ser inhaladas y prvocar reacción de cuerpo extraño, se deben incluir algunas, que forman parte de fórmulas en la preparación de biberones (13).

Los dos casos, en que se demostró aspiración de Ceratonia siliqua, componente del Arobón ( $\mathrm{R}$ ), prducto de distribución mundial, dieron lugar a cuadros clínicos susceptibles de ser interpretados como Neumopatías crónicas. Uno de ellos se presentó como un síndrome bronquial obstructivo crónico irreversible y el otro con los caracteres clínico - radiológicos de una Neumonitis crónica. En ninguno de ellos, los exámenes habitualmente indicados para esclarecer su etiología permitieron avanzar en este sentido, y fue sólo la biopsia pulmonar, que detectó el agente etiológico.

En este caso se trata de material de origen vegetal y que en la observación directa se caracteriza por cuerpos de forma y tamaño variables, de color pardo oscuro, gran densidad óptica, muchos de los cuales presentan birrefringencia y extremos aguzados (Fig. 6). Estas características se conservan en el tejido pulmonar afectado, que presenta granulomas con células gigantes de cuerpo extraño, fibrosis intersticial y, en ocasiones, áreas de inflamación crónica linfomatosa.

La reacción inflamatoria del tejido, a distancia de las partículas visibles microscópicamente, sugiere algún mecanismo de solubilización y transporte. La gran cantidad de calcio en el polvo de Ceratonia siliqua ( 350 mgr. \%) permite suponer, que la causa de

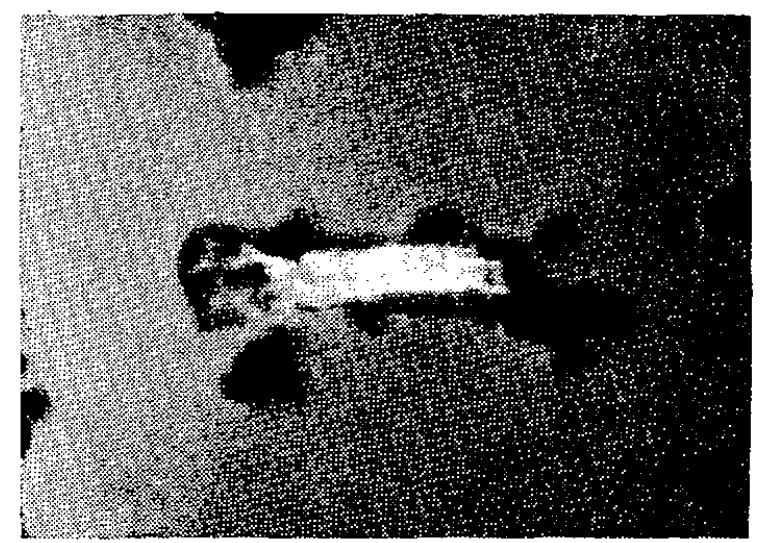

6. Microfotografía de partícula de polvo de Ceratonia siliqua. Luz polarizada $100 \mathrm{X}$. la irritación puede ser algún compuesto cálcico poco soluble.

En nuestros casos, el control alejado, entre 1-3 años demostró la persistencia de manifestaciones clínico - radiológicas, sin obtenerse mayores beneficios con la terapia esteroidal, lo que podría sugerir la instalación de una Neumopatía proliferativa, de curso eventualmente progresivo.

En la actualidad desconocemos la verdadadera incidencia de esta etiología en las Neumopatías crónicas de la infancia. Sin embargo cabe destacar, por una parte, la gran frecuencia de la insuficiencia gastro-esofágica, particularmente en lactantes menores de 1 año, y por otra, la comunicación en un lapso relativamente breve, de 4 necropsias, en los que se detectó esta etiología (13).

$\mathrm{Su}$ especificidad ha sido confirmada mediante la contraprueba, inyectando esta sustancia en suspensión a lóbulos pulmonares normales y por el examen microscópico directo del preparado (13).

El conocimiento de la severidad de este cuadro clínico e histológico, de origen iatrogénico, nos parece, que deberá condicionar, por parte del pediatra, una ponderación muy cuidadosa, frente a la indicación de una terapéutica dietética enteramente prescindible y reemplazada por otras medidas, que además de este riesgo involucra una interferencia en la absorción del nitrógeno, como se ha demostrado en trabajos recientes (8).

\section{RESUMEN}

Se presentan 3 casos de Neumopatia crónica en lactantes: una debido a aspiración de vaselina y dos a Ceratonia siliqua (Arobón) (R).

En los 3 casos destacó clínicamente: polipnea de reposo persistente, signologia de condensación en uno y bronquial en los dos casos secundarios a aspiración de Arobón (R).

Radiológicamente predominaron las sombras difusas, localizadas de preferencia en el L. S. D. y L. M., de carácter persistente.

El mecanismo aspirativo se relacionó en el caso de la Neumonía lipoidea con el reflujo por insuficiencia gastro-esofágica.

La confirmación etiológica se obtuvo en los tres casos mediante la biopsia pulmonar a cielo abierto.

El seguimiento clínico-radiológico en lapsos variables entre 1-3 años, permitió confirmar la persistencia de las lesiones, que no se beneficiaron con la terapia esteroidal prolongada. 
Se comentan los mecanismos etiopatogénicos, las alteraciones histopatológicas y el pronóstico de estas Neumopatías.

Se señala el riesgo de ciertas substancias medicamentosas o dietéticas en relación a su papel etiológico en Neumopatías crónicas de la infancia, favorecidas por los mecanismos aspirativos tan propios de esta edad.

\section{SUMMARY}

3 cases of chronic pulmonary disease in infants are presented. One of them due to aspiration of vaseline and the other two produced by Ceratonia siliqua (Arobón (R) ).

The 3 patients presented persistent tachypnea at rest, the signology was of consolidation in one of them and bronchial in the other two secondary to aspiration of Arobón (R).

$\mathrm{X}$. rays films showed persistent, predominantly diffuse shadows, preferently localized to right upper and middle lobes.

The aspiration in the case of lipid pneumonia was related with reflux due to gastroesophageal insufficiency.

The etiology was corroborated by lung biopsy performed through open thoracotomy.

Clinical - radiologic follow - up was made at variable intervals, from 1 to 3 years demonstrating persistence of the lesions without improvement in spite of prolonged esteroid therapy.

Etiopathogenetic mechanisms, histological alterations and prognosis in these pneumonias are comented.

The hazard of certain medicinal or dietetical substances is noted as etiologic factors of chronic pneumonias in infancy, which are favored my aspiration mechanisms, characteristics at this age.

\section{REFERENCIAS}

1.- Adams, W. C.: En Kendig E. L. Disorders of the Respiratory tract in children; Saunders, Philadelphia, 1967.

2.-Avery M. E., Fletcher B. D.: The lung and its disorders in the Newborn infant. Saunders; Philadelphia, 1974.
3.-Baum G. L.: Textbook of pulmonary diseases. Little Brown and Co. Boston, 1965.

4.-- Casar C., Ceruti E., Díaz A.: Bronquitis obstructiva recidivante del lactante. Rev. Chil. Pediat. 44: $337,1973$.

5.-Crofton J.: Enfermedades respiratorias. Marin S. A., Barcelona, 1971.

6.-De Carlo: Citado por Avery (ref. No 2).

7.-Gerberaux J., Bauchard A., Grunberg J., Tournier G. Gaultier Cl.: Bronchopneumopaties repetées et reflux gastro - oesophagic. Annals de Pédiatrie 48: 2323, 1972.

8.-Martner J. G., Jarpa S., Biancani F.: Estudio comparativo entre una leche semi-descremada y otra similar con agregado de harina de algarroba. Rev. Chil. Pediat. 44: 407, 1973.

9.-Matsaniotis N., Karpouzas J., Tzortzatow, Vallianou and Tsagousnis E.: Aspiration due to difficulty in swallowing. Arch. Dis. Child. 46: 788, 1971.

10.- McNamara J. J., Paulson D. L., Urschel H. C.: Hiatal hernia and gastrooesophageal reflux in children. Pediatrics 43: 527, 1969.

11.-Milton V. Davis: Relationship between pulmonary disease, hiatal hernia and gastro-oesophageal reflux. N. Y. State J. of Med. April 15: 935, 1972.

12.- Naclerio E. A.: Bronchopulmonary Diseases. Hoebert Harper, New York, 1959.

13.- Parraguez J., Chuaqui B.: Bronconeumonía por aspiración de material extraño con fécula de Ceratonia siliqua. Inédito.

14.- Singleton E. B., Wagner M. L.: Atlas radiológico de anomalías pulmonares en el niño. Ed. Pediátrica, Barcelona, 1972.

15.- Wesenberg R. L.: The newborn chest. Harper and Row, New York, San Francisco, London, 1973. 